\title{
Growth of Volume in Fatou-Bieberbach Regions
}

By

\author{
Jean-Pierre RosAY ${ }^{*}$ and Walter RUDIN*
}

\section{Introduction}

We call a set $\Omega \subset C^{n}$ a Fatou-Bieberbach region (F.B. region, for brevity) if $\Omega$ is the range of a one-to-one holomorphic map $\Phi$ with domain $C^{n}$, and $\Omega \neq C^{n}$.

In other words, F. B. regions are proper subsets of $\boldsymbol{C}^{n}$ that are biholomorphically equivalent to $C^{n}$. Numerous examples of such regions (when $n>1$ ) can be found in [2] and [6].

It is easy to see that the volume (i.e., the $2 n$-dimensional Lebesgue measure) of every F. B. region is infinite, simply because

$$
\operatorname{vol}(\Omega)=\int_{C^{n}}|J \Phi(z)|^{2} d m_{2 n}(z) .
$$

Here $J \Phi$ is the complex Jacobian of $\Phi[7 ; \mathrm{p} .11]$, hence $J \Phi$ is holomorphic, $|J \Phi|^{2}$ is subharmonic and of course positive, and its integral over $C^{n}$ is therefore infinite.

Let $B$ be the open unit ball of $C^{n}$. Thus $r B$ is the ball of radius $r$, centered at 0 . The preceding paragraph shows that $\operatorname{vol}(\Omega \cap r B)$ must tend to $\infty$ as $r \rightarrow \infty$, whenever $\Omega$ is F.B. Theorem 1 of the present paper shows that this can happen arbitrarily slowly.

We became interested in $\operatorname{vol}(\Omega \cap r B)$ because we wanted to know (we still don't) whether there is an F.B. region in $C^{2}$ that does not intersect the set $\{z w=0\}$, a union of two intersecting complex lines. (This is a special case of a more general question: Which analytic varieties $V$ can be avoided by F.B. regions? When $V$ is a complex line in $C^{2}$ then it can be done; see [3] or Example 9.7 in [6]. In this direction, Bedford and Smillie [1] proved an interesting result concerning algebraic varieties.) If there were such a region, it would be the range of a biholomorphic $\Phi$ of the form

Communicated by K. Saito, August 3, 1992.

1991 Mathematics Subject Classification: Primary 32H02.

* University of Wisconsin-Madison, Madison, WI 53706, USA.

${ }^{1}$ Partially supported by an NSF Grant.

Key Words: Biholomorphic maps, Fatou-Bieberbach regions. 


$$
\Phi(z, w)=(\exp 2 \pi i f(z, w), \exp 2 \pi i g(z, w))
$$

where $f, g: C^{2} \rightarrow C$ are entire. Put $\Psi=(f, g)$. Then $\Omega_{0}=\Psi\left(C^{2}\right)$ is an F. B. region with the following very special geometric property:

If $(z, w) \in \Omega_{0}$ and $m, n$ are integers, not both 0 , then $(z+m, w+n)$ is not in $\Omega_{0}$.

(Otherwise, $\Phi$ would not be one-to-one.) A weak consequence of this is that for every choice of $\left(y_{0}, v_{0}\right) \in R^{2}$ the plane

$$
\left\{(z, w): \operatorname{Im} z=y_{0}, \operatorname{Im} w=v_{0}\right\}
$$

intersects $\Omega_{0}$ in a set whose area is $\leqq 1$. This leads to

$$
\operatorname{vol}\left(\Omega_{0} \cap r B\right)=O\left(r^{2}\right)
$$

which is much smaller than the trivial estimate $O\left(r^{4}\right)$.

So, if we could have shown that $O\left(r^{2}\right)$ cannot be achieved by any F. B. region in $C^{2}$, we would have proved that $\{z w=0\}$ cannot be avoided.

An earlier result (Example 9.5 in [6]) gave an F. B. region $\Omega \subset C^{2}$ and a vector $v \in C^{2}$ such that the regions $\Omega+k v(k=0, \pm 1, \pm 2, \cdots)$ are disjoint. Therefore

$$
\operatorname{vol}(\Omega \cap r B)=O\left(r^{3}\right)
$$

for this particular $\Omega$.

Theorem 1. If $n>1$ and $\mu:[0, \infty) \rightarrow[0, \infty)$ satisfies $\mu(r) \rightarrow \infty$ as $r \rightarrow \infty$, then there exists a holomorphic one-to-one map $F: C^{n} \rightarrow C^{n}$, with $J F \equiv 1$, whose range $\Omega$ satisfies

$$
\lim _{r \rightarrow \infty} \frac{\operatorname{vol}(\Omega \cap r B)}{\mu(r)}=0 .
$$

We emphasize " $J F \equiv 1$ " because there exist F. B. regions in every $\boldsymbol{C}^{n}$ (with $n>1$ ) which are not the range of any volume-preserving biholomorphic map with domain $C^{n}$. ([4], [5], or [6], Remark 6.5 and p. 77).

By. a cube in $C^{n}$ we shall mean the Cartesian product of $n$ equal squares in $C$ whose sides are parallel to the real and imaginary axes.

Theorem 1 is an immediate consequence of Theorem 2. We only need to place the cubes $K_{j}$ so that their centers tend to $\infty$ sufficiently rapidly.

Theorem 2. Let $n>1$. Let $K_{j}(j=0,1,2, \cdots)$ be disjoint compact unit cubes in $C^{n}$, centered at points $\left(t_{j}, \cdots, t_{j}\right) \in R^{n}$.

To every $\varepsilon>0$ ocrresponds then a one-to-one holomorphic map $F: C^{n} \rightarrow C^{n}$, with $J F \equiv 1$, and an open set $Z \subset C^{n}$ with $\operatorname{vol}(Z)<\varepsilon$, such that 


$$
F\left(\boldsymbol{C}^{n}\right) \subset Z \cup \bigcup_{j=1}^{\infty} K_{j}
$$

For simplicity, we shall write the proof of Theorem 2 for the case $n=2$. The place where this helps is the last part of the proof. There we use shears in two (complex) directions. In $C^{n}$ we would have to use $n$ directions and thus would have to take many more steps, but the principle is exactly the same. This last part of the proof (the Proposition) is in fact very similar to part of the proof of Lemma 7.2 in [6], but the differences are significant enough that it seems best to include the details.

The following one-variable lemma will be used in the proof of the Proposition at the end of this paper.

Lemma. Suppose that $\left\{A_{i}: i=1,2,3, \cdots\right\}$ is a disjoint collection of compact convex sets in $C$ such that no bounded set intersects infinitely many $A_{i}$ 's. If, for all $i \geqq 1, g_{i}$ is holomorphic in a neighborhood of $A_{i}$, and $\tau_{i}>0$, then there exists an entire function $f: C \rightarrow C$ such that

$$
\left|f(z)-g_{i}(z)\right|<\tau_{i}
$$

for all $z \in A_{\imath}, \quad i=1,2,3, \cdots$.

This can be proved by repeated applications of Runge's Theorem, followed by a passage to the limit.

In the proof of the Proposition we will be dealing with collections of compact cubes with disjoint projections. These cubes will be slightly distorted, but the distortions will be kept so small that the convex hulls of their projections remain disjoint. The $A_{i}$ 's of the Lemma will be these convex hulls; the $g_{i}$ 's will be constants.

Proof of Theorem 2. $C^{2}$ is the union of concentric open cubes $Q_{n}$, so chosen that $\bar{Q}_{0} \subset K_{0}$ and $\bar{Q}_{n} \subset Q_{n+1}$ for all $n \geqq 0$. Let $Q_{-1}=0$.

The collection $\mathcal{E}=\left\{K_{0}, K_{1}, K_{2}, \cdots\right\}$ contains disjoint subcollections $\mathcal{E}_{n}$ such that $\mathcal{E}_{0}=\left\{K_{0}\right\}, \mathcal{E}_{n}$ is infinite for $n \geqq 1$, and the union $E_{n}$ of the members of $\mathcal{E}_{n}$ does not intersect $\bar{Q}_{n+1}$.

The desired map $F$ will carry practically all of $Q_{n} \backslash Q_{n-1}$ into $E_{n}$.

Assume $\varepsilon<1 / 100$ and pick $\varepsilon_{n}>0$ so that $\varepsilon_{0}+\varepsilon_{1}+\varepsilon_{2}+\cdots<\varepsilon$.

Define $F_{0}(z) \equiv z, Y_{0}=\emptyset$, and consider the following induction hypotheses $H(n)$ :

$\left(a_{n}\right) \quad F_{n} \in \operatorname{Aut}\left(C^{2}\right), J F_{n} \equiv 1$, and

$$
\left|F_{n}(z)-F_{n-1}(z)\right|<\varepsilon_{n} \quad \text { on } \bar{Q}_{n-1} .
$$

$\left(b_{n}\right)$ For $j=0,1, \cdots, n$ there are open sets $Y_{j} \subset Q_{j}$, vol $\left(Y_{j}\right)<\varepsilon_{j}$, such that, setting

$$
X_{j}=Q_{j} \backslash\left(Q_{j-1} \cup Y_{j}\right)
$$


the set $F_{n}\left(X_{j}\right)$ lies in the interior of $E_{j}$.

$\left(c_{n}\right)$ If $j>n$ and $z \in E_{j}$ then

$$
\left|F_{n}(z)-z\right|<\varepsilon_{0}+\varepsilon_{1}+\cdots+\varepsilon_{n} .
$$

These hold for $n=0$.

We will see that $H(n)$ implies $H(n+1)$. Once this is done, the theorem follows almost immediately:

By $\left(a_{n}\right),\left\{F_{n}\right\}$ converges, uniformly on compact subsets of $C^{2}$, to a one-toone holomorphic map $F$ with $J F \equiv 1$. Then $\left(b_{n}\right)$ implies that $F\left(X_{\jmath}\right) \subset E$, for all $j$. Setting $Y=\cup Y_{j}$ we see that $\operatorname{vol}(Y)<\varepsilon, Y$ is open, and

$$
F\left(\boldsymbol{C}^{2} \backslash Y\right) \subset \bigcup_{j=0}^{\infty} F(X) \subset \bigcup_{j=0}^{\infty} E_{\jmath} \subset \bigcup_{i=1}^{\infty} K_{\imath} .
$$

Since $F\left(\boldsymbol{C}^{2} \backslash Y^{Y}\right)=F\left(\boldsymbol{C}^{2}\right) \backslash F(Y)$ and $\operatorname{vol}\left(F\left(Y^{*}\right)\right)=\operatorname{vol}\left(Y^{r}\right)<\varepsilon$, we are done, setting $F(Y)=Z$.

We turn to the induction step. Assume $H(n)$.

Since $F_{n}\left(X_{\jmath}\right)$ is a compact subset of the interior of $E_{\jmath}$, there exists $\rho_{n}, 0<$ $\rho_{n}<\varepsilon_{n+1}$, such that

$$
\operatorname{dist}\left(F_{n}\left(X_{\jmath}\right), C^{2} \backslash E_{\jmath}\right)>\rho_{n} \quad(0 \leqq j \leqq n) .
$$

There exists $\delta_{n}>0$ such that

$$
\left|F_{n}\left(z^{\prime}\right)-F_{n}(z)\right|<\rho_{n} \quad \text { if } z \in \bar{Q}_{n},\left|z^{\prime}-z\right|<\delta_{n} .
$$

For $j>n$, let $K_{j s}(s=1,2,3, \cdots)$ be the members of $\mathcal{E}_{\jmath}$. There exist $\delta_{n j s}>0$ such that

$$
\left|F_{n}\left(z^{\prime}\right)-F_{n}(z)\right|<\varepsilon_{n+1} \quad \text { if } z \in K_{\jmath s},\left|z^{\prime}-z\right|<\delta_{n \jmath s} .
$$

We now claim the following.

Proposition. If $H(n)$ holds then there exists $\Phi_{n} \in \operatorname{Aut}\left(C^{2}\right)$, with $J \Phi_{n} \equiv 1$, and there exists an open set $Y_{n+1} \subset Q_{n+1}$ with $\operatorname{vol}\left(Y_{n+1}\right)<\varepsilon_{n+1}$, such that

$$
\begin{aligned}
& \left|\Phi_{n}(z)-z\right|<\delta_{n} \quad \text { if } z \in \bar{Q}_{n}, \\
& \left|\Phi_{n}(z)-z\right|<\delta_{n \jmath s} \quad \text { if } z \in K_{j s}, j>n, \\
& \Phi_{n}\left(Q_{n+1} \backslash\left(Q_{n} \cup Y_{n+1}\right)\right) \subset F_{n}^{-1}\left(E_{n+1}\right) .
\end{aligned}
$$

Once this is proved, put $F_{n+1}=F_{n} \circ \Phi_{n}$. Then (12) and (10) show that

$$
\left|F_{n+1}(z)-F_{n}(z)\right|<\rho_{n}<\varepsilon_{n+1} \quad \text { if } z \in \bar{Q}_{n} .
$$

This gives $\left(a_{n+1}\right)$.

If $j \leqq n$ and $z \in X$, then (note that $X_{j} \subset \bar{Q}_{n}$ ) (15) and (9) show that $F_{n+1}(z)$ 
$\in E_{\jmath}$. Thus $F_{n+1}\left(X_{\jmath}\right) \subset E_{j}$ for $j \leqq n$, and then (14) gives $F_{n+1}\left(X_{n+1}\right) \subset E_{n+1}$, which completes $\left(b_{n+1}\right)$.

If $j>n$ and $z \in E_{\mathrm{j}}$ then (13), (11), and $\left(c_{n}\right)$ imply $\left(c_{n+1}\right)$.

So all that remains to be done is to give the

Proof of the Proposition. $\Phi_{n}$ will be obtained as a composition of 4 shears : $\Phi_{n}=\sigma_{1} \circ \sigma_{3} \circ \sigma_{2} \circ \sigma_{1}$.

Define $\pi_{1}(z, w)=z, \quad \pi_{2}(z, w)=w$. Recall that the projections $\pi_{1}\left(K_{j}\right)$ and $\pi_{2}\left(K_{j}\right)$ are lined up along the real axis in $C$.

Fix $n$. Then $Q_{n}=S \times S, Q_{n+1}=T \times T$, where $S, T$ are concentric open squares in $C, \bar{S} \subset T$. Put finitely many disjoint closed squares $\gamma_{m}$ into $T$, in such a way that

(i) the diameter of each $\gamma_{m}$ is $<1 / 100$,

(ii) no $\gamma_{m}$ intersects the boundary of $S$, and

(iii) the union of the cubes $\Gamma_{m k}=\gamma_{m} \times \gamma_{k}$ covers all of $Q_{n+1}$, except for a set $Y_{n+1}$ of volume $<\varepsilon_{n+1}$.

Let $W$ be the collection of all $\Gamma_{m k} \subset Q_{n+1} \backslash Q_{n}$.

Let $W_{1}=\left\{\Gamma_{m k}: \pi_{2}\left(\Gamma_{m k}\right) \subset T \backslash S\right\}$.

The lemma will be tacitly used in the construction of each $\sigma$, to give us certain holomorphic functions $\varphi_{i}: \boldsymbol{C} \rightarrow \boldsymbol{C}$.

Recall that we are assuming $H(n)$. In particular, $\left(c_{n}\right)$ says that $F_{n}$ has moved no point of $E_{j}$ by as much as $\varepsilon$ if $j>n$. Thus

$$
D_{\jmath}=F_{n}\left(E_{\jmath}\right) \quad(j>n)
$$

is a union of slightly distorted unit cubes whose projections are disjoint.

Put $\sigma_{1}(z, w)=\left(z+\varphi_{1}(w), w\right)$, where $\varphi_{1}$ is almost 0 on $\bar{S}$ and on $\pi_{2}\left(D_{\jmath}\right)$ for all $j>n, \varphi_{1}$ is almost equal to a large pure imaginary constant on each $\gamma_{k}$ outside $S$, and these constants are so chosen that the projections $\pi_{1}\left(\sigma_{1}\left(\Gamma_{m k}\right)\right)$, for $\Gamma_{n k} \in W_{1}$, are disjoint from each other and are far from $\pi_{1}\left(\sigma_{1}(\bar{T} \times \bar{S})\right)$ and from $\pi_{1}\left(\sigma_{1}\left(D_{j}\right)\right)$ for all $i>n$.

Put $\sigma_{2}(z, w)=\left(z, w+\varphi_{2}(z)\right)$. Again, $\varphi_{2}$ is almost 0 on $\pi_{1}\left(\sigma_{1}\left(\bar{Q}_{n}\right)\right)$ and on $\pi_{1}\left(\sigma_{1}\left(D_{j}\right)\right)$ for all $j>n, \varphi_{2}$ is almost equal to a large pure imaginary constant on each projection $\pi_{1}\left(\sigma_{1}\left(\Gamma_{m_{k}}\right)\right)$, this time for all $\Gamma_{m k} \in W$, and these constants are so chosen that the projections $\left(\pi_{2^{\circ}} \sigma_{2} \circ \sigma_{1}\right)\left(\Gamma_{m k}\right)$ are disjoint from each other and are far from $\left(\pi_{2} \circ \sigma_{2} \circ \sigma_{1}\right)\left(\bar{Q}_{n}\right)$ and from $\left(\pi_{2} \circ \sigma_{2} \circ \sigma_{1}\right)\left(D_{\jmath}\right)$ for all $j>n$.

Setting $\Gamma_{m k}^{\prime}=\left(\sigma_{2} \circ \sigma_{1}\right)\left(\Gamma_{m k}\right), Q_{n}^{\prime}=\left(\sigma_{2} \circ \sigma_{1}\right)\left(Q_{n}\right)$, and $D_{j}^{\prime}=\left(\sigma_{2} \circ \sigma_{1}\right)\left(D_{\jmath}\right)$ we have now reached the following position:

( $\alpha$ ) $Q_{n}^{\prime}$ is almost the same as $Q_{n}$.

( $\beta$ ) The sets $Q_{u}^{\prime}, \Gamma_{m k}^{\prime}$ (for $\Gamma_{m k} \in W$ ), and $D_{j}^{\prime}$ (for $j>n$ ) have disjoint $\pi_{2^{-}}$ images. 
( $\gamma$ ) each $\Gamma_{m k}^{\prime}$ differs from a translate of $\Gamma_{m k}$ (a cube of edge $<1 / 100$ ) by a very small distortion, and

$(\delta)$ each component of $D_{j}^{\prime}$ is a small distortion of one of the given unit cubes and therefore contains a cube $C \subset E_{j}$ of edge $1 / 2$. (Recall $\left(c_{n}\right)$.)

Put $\sigma_{3}(z, w)=\left(z+\varphi_{3}(w), w\right)$, where $\varphi_{3}$ is almost 0 on $\pi_{2}\left(Q_{n}^{\prime}\right)$ and on $\pi_{2}\left(D_{\jmath}^{\prime}\right)$ for $j>n$, and is almost constant on each $\pi_{2}\left(\Gamma_{m k}^{\prime}\right)$, and these constants are so chosen that $\sigma_{3} \circ \sigma_{2} \circ \sigma_{1}$ moves the center of each $\Gamma_{m k} \in W$ to the center of $\pi_{1}\left(C_{m k}\right)$, where $C_{m k}$ is one of the cubes $C$ as in $(\delta)$ above, in $D_{n+1}^{\prime}$.

Finally, $\sigma_{4}(z, w)=\left(z, w+\varphi_{4}(z)\right)$, where $\varphi_{4}$ is almost 0 on $\pi_{1}\left(\sigma_{3}\left(Q_{n}^{\prime}\right)\right)$ and on $\pi_{1}\left(\sigma_{3}\left(D_{j}^{\prime}\right)\right)$ for $j>n$, and $\varphi_{4}$ is almost constant on each $\pi_{1}\left(\sigma_{3}\left(\Gamma_{m k}^{\prime}\right)\right)$, and these constants are so chosen that each $\sigma_{4}\left(\sigma_{3}\left(\Gamma_{m k}^{\prime}\right)\right)$ lies in one of the above-mentioned cubes $C_{m k}$ in $D_{n+1}^{\prime}$.

If all approximations implicit in "almost" are sufficiently close, then $\Phi_{n}=$ $\sigma_{1} \circ \sigma_{3} \circ \sigma_{2} \circ \sigma_{1}$ will satisfy (12), (13), and (14).

\section{References}

[1] Bedford, E. and Smillie, J., Fatou-Bieberbach domains arising from polynomial automorphisms, Indiana U. Math. J., 40 (1991), 789-792.

[2 $]$ Dixon, P.G. and Esterle, J., Michael's problem and the Poincaré-Fatou-Bieberbach phenomenon, Bull. AMS N. S., 15 (1986), 127-187.

[3] Nishimura, Y., Automorphismes analytiques admettant des sous variétés de points fixes dans une direction transversale, J. Math. Kyoto Univ., 23 (1983), 289-299.

[4] - Applications holomorphes injectives de $C^{2}$ dans lui-même qui exceptent une droite complexe, J. Math. Kyoto Univ., 24 (1984), 755-761.

[5] —, Applications holomorphes injectives à Jacobien constant de deux variables, J. Math. Kyoto Univ., 26 (1986), 697-709.

[6] Rosay, J.-P. and Rudin, W., Holomorphic maps from $\boldsymbol{C}^{n}$ to $\boldsymbol{C}^{n}$, Trans. AMS, 310 (1988), 47-86.

[7] Rudin, W., Function Theory in the Unit Ball of $\boldsymbol{C}^{n}$, Springer-Verlag, 1980. 